\title{
Stage III Bone Cancer AJCC v8
}

National Cancer Institute

\section{Source}

National Cancer Institute. Stage III Bone Cancer A/CC v8. NCI Thesaurus. Code C136619.

Stage III includes: T3, NO, M0, G2 or G3. T3: Discontinuous tumors in the primary bone site. NO: No regional lymph node metastasis. MO: No distant metastasis. G2: Moderately differentiated, high grade. G3: Poorly differentiated, high grade. (AJCC 8th ed.) 